\title{
Ionized gas outflows and global kinematics of low-z luminous star-forming galaxies
}

\author{
S. Arribas ${ }^{1}$, L. Colina ${ }^{1}$, E. Bellocchi ${ }^{1}$, R. Maiolino ${ }^{2}$ and \\ M. Villar-Martín ${ }^{1}$ \\ ${ }^{1}$ CSIC - Departamento de Astrofísica-Centro de Astrobiología (CSIC-INTA),Torrejón de \\ Ardoz, Madrid, Spain \\ email: arribas@cab.inta-csic.es \\ ${ }^{2}$ Cavendish Laboratory, University of Cambridge 19 J. J. Thomson Avenue, Cambridge CB3 \\ 0HE, UK and Kavli Institute for Cosmology, University of Cambridge, Madingley Road, \\ Cambridge CB3 0HA, UK
}

\begin{abstract}
We have studied the kinematic properties of the ionized gas outflows and ambient interstellar medium (ISM) in a large and representative sample of local U/LIRGs (58 systems, 75 galaxies) at galactic and sub-galactic (i.e.,star-forming clumps) scales, thanks to integral field spectroscopy (IFS)-based high signal-to-noise integrated spectra. The main results have been recently presented in Arribas et al. (2014), and are briefly summarized here.
\end{abstract}

Keywords. - galaxies: evolution - galaxies: ISM -galaxies: Outflows

\section{Introduction}

The role of outflows governing galaxy evolution is believed to be crucial, as they can regulate and quench both $\mathrm{SF}$ and black hole activity, being also the primary mechanism by which dust and metals are redistributed over large scales within the galaxy, or even expelled into the intergalactic medium (IGM, see Veilleux, Cecil, \& Bland-Hawthorn 2005 for a review). Hence most of the models of galaxy formation require energetic outflows to reproduce the observed properties (e.g., Silk and Rees 1998; Di Matteo et al. 2005; Hopkins et al.2012). Although the general theoretical framework seems reasonably well established, it is not well constrained by observations as it is unclear how the dynamical status of the ISM and the properties of the outflows depend on the galaxy mass, largescale tidal forces, SFR, and/or type and luminosity of the AGN.

We have studied the main integrated kinematic properties of the ionized interstellar medium in the largest sample of local (U)LIRGs observed via IFS (Arribas et al. 2014). Specifically, we have focussed the study on i) the global dynamical status of the ambient ionized gas via the velocity dispersion of the ISM, and ii) the properties of the ionized gas outflows, and how they relate to the characteristics of the system like the global SFR, SF density, morphological merging phase, and the presence of AGNs. For the present study, we follow a methodology often used at high-z, which consists in integrating the IFS spectra for deriving the (flux weighted) average properties of the system. This allow us a direct comparison with high-z samples. The properties of a sample of luminous SF clumps have also been investigated.

\section{Main results and conclusions}

A summary of the main results are given below (see Arribas et al. 2014 for details).

(a) Global dynamical status of the ambient ionized gas 
- The role of star formation regulating $\sigma$. Isolated non-AGN LIRG discs have velocity dispersions of the ambient ionized gas $\left(45 \pm 4 \mathrm{kms}^{-1}\right)$ about a factor 2 larger than in less active spirals, likely as a consequence of the increased star formation. However, the heating effects of the star formation tend to saturate in the U/LIRGs luminosity range as indicated by the weak dependency of the velocity dispersion on the IR-derived SFR $\left(\sigma \propto \operatorname{SFR}\left(L_{I R}\right)^{+0.12}\right)$. The velocity dispersion also has a very slight dependency on the $\mathrm{H} \alpha$ star formation surface density $\left(\sigma \propto \Sigma_{S F R}^{0.06}\right)$, departing significantly from the expected behaviour if energy release by the starburst were regulating $\sigma$ (i.e., $\sigma \propto \Sigma_{S F R}^{0.5}$ ) (see Fig.1). The relatively small role of the star formation driving the dynamical status of the ambient ionized gas in U/LIRGs is reinforced by the fact that the clumps of strong SF do not have an increase in velocity dispersion associated, as we find in the corresponding IFS maps.

- Heating by tidal forces. The $\sigma$ of the ionized gas follows a sequence of increasing values with the dynamical phase of the system, from isolated discs to interacting pairs and mergers ( $\langle\sigma\rangle$ of 45,59 , and $73 \mathrm{~km} \mathrm{~s}^{-1}$, respectively). This evolution towards dynamically hotter systems seems to be mainly driven by the gravitational energy release associated with the interacting/merging process, rather than with SF.

- The role of AGNs. The impact of AGNs in the dynamical status of the ionized gas is significant in ULIRGs, with a net increase in the average velocity dispersion from $74 \pm 6 \mathrm{~km} \mathrm{~s}^{-1}$ for non-AGNs to $109 \pm 14 \mathrm{~km} \mathrm{~s}^{-1}$ for AGNs, i.e., by a factor 1.5 . For LIRGs the impact is significantly lower (i.e., a factor of 1.2).

- Local versus high-z SF galaxies. The low-z U/LIRGs cover a range in $\sigma$ and $\Sigma_{S F R}$ similar to that of high-z SFGs. Moreover, the observed weak dependency of $\sigma$ of the ionized gas on the compactness of the SF for local U/LIRGs $\left(\sigma \propto \Sigma_{S F R}^{n}\right.$, with $n=0.06 \pm 0.03)$ is in very good agreement with the dependency reported by Genzel et al. (2011) at high-z $(n=0.07 \pm 0.025)$ (Fig.1).

- SF clumps properties and scaling laws. The sizes, luminosities and velocity dispersions of the clumps are in general consistent with the mean scaling relations derived for (mainly) giant HII regions in spirals, although the smaller clumps (diameter less than $1 \mathrm{kpc}$ ) appear more luminous, at a given size. When compared with high-z clumps, they are in general smaller, less luminous and have lower $\sigma$. However, part of these differences could be due to systematic observational effects.

(b) Ionized gas outflows

- Detection rate. The presence of ionized gas outflows in U/LIRGs seems to be universal based on the detection of a secondary broad, usually blueshfited, kinematic component in the $\mathrm{H} \alpha$ emission line.

- The relative role of $S F$ and $A G N$. In non-AGN U/LIRGs, the maximum velocity of the outflow $\left(\mathrm{V}_{\max }\right)$ shows a dependency on the $\mathrm{SFR}$ of the type $\mathrm{V}_{\max }$ (non $A G N) \propto S F R^{+0.24}$. In U/LIRGs with an AGN, the velocity increases by a factor of $\sim 2$ with respect to non-AGNs, independent of the global SFR (i.e., $\mathrm{V}_{\max }(A G N) \propto$ $\left.S F R^{+0.23}\right)$. Moreover, the fraction of $\mathrm{H} \alpha$ flux associated with the outflow increases by a factor of $\sim 1.4$ in U/LIRGs with an AGN. Thus, AGNs in U/LIRGs are able to generate faster and more massive ionized gas outflows than pure starbursts.

- Dependency on merger evolution. The observed differences in the properties of the outflows for isolated discs, interacting systems, and mergers are consistent with their associated change in SF. Therefore, the release of gravitational potential along the merger evolution does not seem to affect the properties of the outflow, except for its effects on the SF.

- Outflowing mass and mass loading factors. The outflowing mass rate covers a wide range from about 0.1 to $100 \mathrm{M}_{\odot} y r^{-1}$ with a close to linear dependency with the 


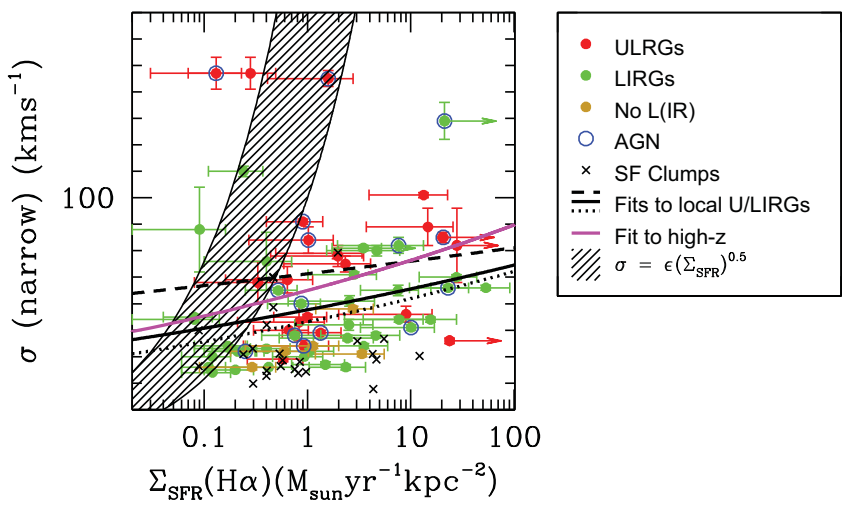

Figure 1. Velocity dispersion of the ISM (narrow component) as a function of the star formation density, $\Sigma_{S F R}$, derived considering the extinction corrected $\mathrm{H} \alpha$ fluxes (see text), and $\mathrm{H} \alpha$ half-light radii (Arribas et al. 2012). The symbol and line codes are indicated in the right panel.The black continuous line corresponds to an unweighted regression to all the U/LIRGs $\left(\sigma \propto \sum_{S F R}^{n}, n=0.06 \pm 0.03, \mathrm{r}=0.3\right)$, while the dotted and dashed lines are for non-AGNs (n $=0.06, \mathrm{r}=0.4)$ and AGNs $(\mathrm{n}=0.03, \mathrm{r}=0.1)$, respectively. The shaded region corresponds to a behaviour of the type $\sigma\left(\mathrm{kms}^{-1}\right)=\epsilon \Sigma_{S F R}^{0.5}$ for efficiencies $(\epsilon)$ between 100 and 240 , which is expected for SF driven motions (Lehnert et al. 2009). The magenta line corresponds to the unweighted) fit, found by Genzel et al. (2011), to a large compilation of high-z galaxies and clumps (see text)

IR-derived SFR $\left(\dot{M}_{g} \propto S F R^{+1.11}\right)$. Mass loading factors $(\eta)$ are in general below one, with only a few AGNs above this limit. The dependency of $\eta$ on the dynamical mass of the system agrees well with recent simulations (Hopkins et al. 2012). However, the observed absolute values for $\eta$, as well as its trend with star formation surface density $\left(\left(\eta \propto \Sigma_{S F R}^{+0.21}\right.\right.$, for all non-AGN U/LIRGs) disagree with these models.

- Escape fractions and IGM metal enrichment. Only less massive $\left(\log \left(\mathrm{M}_{d y n} / M_{\odot}\right)\right.$ $<$ 10.4) U/LIRGs have outflowing maximum velocities $\left(\mathrm{V}_{\text {max }}\right)$ higher than their escape velocities, and therefore part of their ejected metal-rich gas could escape the gravitational potential of the host galaxy. More massive galaxies $\left(\log \left(\mathrm{M}_{d y n} / M_{\odot}\right)>\right.$ 10.4) would retain all the gas, even if they host an AGN. These results are consistent with chemical evolutionary models (Fig.2).

- Outflows in low- and high-z star-forming galaxies. The observed average properties (line width, velocity shift, and broad-to-narrow line flux ratio) are similar in low-z U/LIRGs and high-z star-forming galaxies, with hints that the fraction of $\mathrm{H} \alpha$ flux in the broad component could somehow be larger in low-z U/LIRGs. While high-z outflows show mass loading factors above one for galaxies with star formation surface densities above $1 M_{\odot} y r^{-1} k p c^{-2}$, such a threshold is not observed in low-z U/LIRGs even after considering the different gas fraction.

- Outflows in luminous star-forming clumps. Ionized gas outflows appear to be very common (detection rate over 80\%) in the bright SF clumps found in LIRGs, which are characterized by a median star formation surface density of $0.13 M_{\odot} y r^{-1} k p c^{-2}$ and half-light radius of $0.49 \mathrm{kpc}$. Their properties (line width, velocity shift, and fraction of $\mathrm{H} \alpha$ flux in the broad component) appear to be less extreme than those associated with the galaxy, and therefore the energy involved in the outflows associated with individual clumps is (in relative terms) more modest than that for the entire galaxy. - Outflows in low- and high-z SF clumps. Ionized gas outflows generated in SF clumps at all redshifts appear to carry a similar fraction of their $\mathrm{H} \alpha$ luminosity. However, high-z SF clumps launch outflows with much higher velocities, factors 4 


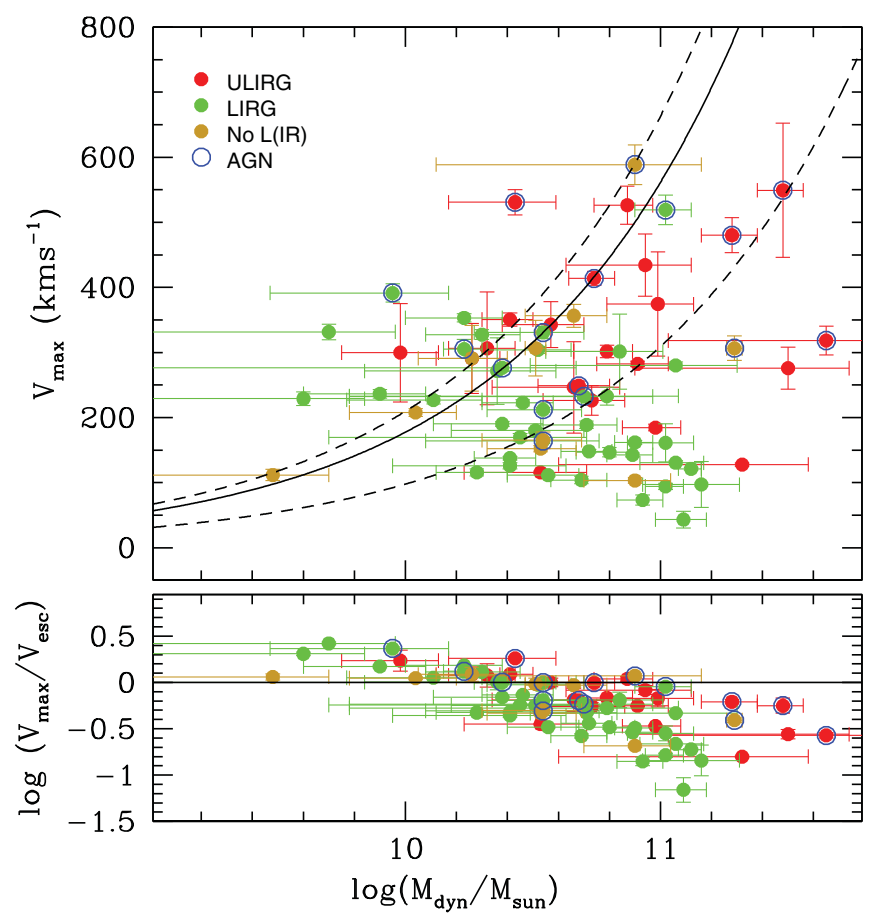

Figure 2. Top panel: Maximum outflow velocity as a function of the dynamical mass. Red and green dots distinguish ULIRGs and LIRGs systems. Orange dots are objects without $\mathrm{L}_{I R}$ determinations. Blue circles indicate objects with evidence of being affected by an AGN in the optical lines (see text). The solid line represents the mean escape velocity for $\mathrm{r}=3 \mathrm{kpc}$, considering a simple model of a truncated isothermal sphere of $\mathrm{r}_{\text {max }} / \mathrm{r}=10$. The dotted lines correspond to values for $\mathrm{r}_{\text {max }} / \mathrm{r}=1$ and 100 (see text). Bottom panel: The maximum outflow velocities are represented in units of the escape velocity for the case $\mathrm{r}_{\text {max }} / \mathrm{r}=10$.

to 10 higher, than clumps in low-z U/LIRGs. For a given observed SF surface density, outflows in high-z clumps would be about one to two orders of magnitude more energetic than the outflows launched by clumps in U/LIRGs, i.e. the most extreme starburst galaxies in our low-z universe.

\section{References}

Arribas, S., Colina, L., Alonso-Herrero, A., et al. 2012, A\&A, 541, A20

Arribas, S., Colina, L., Bellocchi, E., Maiolino, R., \& Villar-Martín, M. 2014, A\&A, 568, A14

Ceverino, D., Dekel, A., \& Bournaud, F. 2010, MNRAS, 404, 2151

Di Matteo, T., Springel, V., \& Hernquist, L. 2005, Nature, 433, 604

Genzel, R., Newman, S., Jones, T., et al. 2011, ApJ, 733, 101

Hopkins, P. F., Quataert, E., \& Murray, N. 2012, MNRAS, 421, 3522

Lehnert, M. D., Nesvadba, N. P. H., Le Tiran, L., et al. 2009, ApJ, 699, 1660

Murray, N., Ménard, B., \& Thompson, T. A. 2011, ApJ, 735, 66

Ostriker, E. C. \& Shetty, R. 2011, ApJ, 731, 41

Silk, J. \& Rees, M. J. 1998, A\&3A, 331, L1

Veilleux, S., Cecil, G., \& Bland-Hawthorn, J. 2005, ARA\&SA, 43, 769 